\title{
Alexitimia y consumo de drogas en hombres y mujeres en tratamiento
}

\author{
Valeriano Raúl García Aurrecoechea', Solveig E. Rodríguez Kuri', Alberto J. Córdova Alcaráz', \\ Carmen Fernández-Cáceres' \\ Centros de Integración Juvenil, A.C., México
}

\begin{abstract}
RESUMEN
Introducción: la alexitimia es un rasgo de la personalidad caracterizado por la dificultad para identificar y expresar emociones, con mayor prevalencia en hombres que en mujeres y presente en la mitad de los consumidores de drogas. Objetivo: comparar la presencia de alexitimia en hombres y mujeres que acuden a solicitar tratamiento por consumo de drogas y explorar diferencias según el tipo de droga por la que se solicita atención (droga de mayor impacto). Método: estudio transversal comparativo por sexo y por droga de consumo. Resultados: la mitad de los 264 participantes presenta rasgos de personalidad alexitímicos, cuatro veces más que la población general, observándose diferencias por sexo únicamente en la dificultad para identificar emociones (mayor entre los hombres). También destaca que no existan diferencias en el nivel de alexitimia por tipo de droga. Discusión: en concordancia con lo reportado por la literatura, se observa una alta proporción de alexitimia entre los usuarios participantes, lo cual es relevante si se considera que los pacientes con este rasgo presentan una particular dificultad para responder a los tratamientos cognitivo conductuales, los cuales constituyen abordajes ampliamente utilizados en el tratamiento de adicciones. Asimismo, destaca la escasa diferencia entre hombres y mujeres respecto a la presencia de este rasgo, en comparación con lo que se observa en la población general, donde este rasgo es claramente mayor entre los hombres. Lo anterior pone en evidencia la necesidad de desarrollar estrategias que contribuyan a mejorar la capacidad introspectiva y de comunicación afectiva de estos pacientes.
\end{abstract}

\begin{abstract}
Introduction: alexithymia is a personality trait characterized by a difficulty to identify and express emotions -with higher prevalence in men than in women- that is present in half of the drug users. Objective: to compare the presence of alexithymia in men and women looking for drug abuse treatment. Method: cross-sectional comparative study by sex and drug used. Results: half of the 264 participants have alexithymic personality traits, four times more than general population. There were observed differences by sex, but only in the difficulty to identify emotions (higher in men). It also highlights that there are differences in the level of alexithymia by types of drug. Discussion: according with the background on the subject, a high proportion of alexithymia is observed among participating, which is important if it is considered that patients with this trait have a particular difficulty to respond to cognitive behavioral treatments, which are approaches widely used in the treatment of drug addiction. It also highlights the little difference in alexithymia among men and women drug abusers, compared to the general population in which this trait is clearly higher among men. This highlights the need to develop strategies to help improve the introspective and affective communication capacity of these patients.
\end{abstract}

Keywords: alexithymia, drug abuse, gender, treatment.

Palabras clave: alexitimia, consumo de drogas, género, tratamiento.

\section{Autor de correspondencia:}

Solveig E. Rodríguez Kuri. Tlaxcala núm. 208, col. Hipódromo, del Cuauhtémoc, C.P. 06100, Ciudad de México. Tel. +52 (55) 55 59 9949 49, ext. 7713. Correo electrónico: solveigrk@hotmail.com y solveig.rodriguez@cij.gob.mx

Recibido: 24 de agosto de 2016

Aprobado: 27 de septiembre de 2016

DOI: $10.28931 /$ riiad.2016.2.03 


\section{INTRODUCCIÓN}

La alexitimia es una característica de la personalidad que afecta la percepción de las emociones; en el caso de los usuarios de drogas, se le debe tener en cuenta para su tratamiento, ya que puede afectar la eficacia de las intervenciones.

Se trata de un constructo desarrollado a partir de la observación clínica de personas que muestran cierta incapacidad para identificar en ellas mismas el componente afectivo de las emociones y por tanto dificultad para expresarlas. El concepto, propuesto originalmente por Sifneos (1973), consta de tres componentes:

A. Una dificultad marcada para describir sentimientos. B. Una ausencia o reducción importante de la fantasía. C. La manifestación del pensamiento operatorio.

Taylor, Bagby y Parker (1997) la definen como un déficit del procesamiento cognitivo y de la regulación de las emociones; y Alonso-Fernández (2011), como un déficit comunicativo emocional asociado con un pensamiento operacional, alta impulsividad y abundantes sensaciones corporales. Se encuentra presente en aproximadamente $10 \%$ de la población, siendo más frecuente entre los hombres (Levant, Hall, Williams, \& Hasan, 2009), con algunos efectos mediadores diferenciados entre los sexos (Gabriel, Untas, Lavner, Koleck, \& Luminet, 2016); así, las mujeres alexitímicas tienden a ser menos extrovertidas (Günther, Matthes, Kersting, Egloff, \& Suslow, 2016).

No se incluye como categoría diagnóstica en los manuales, más bien se trata de una caracterización del tipo de pensamientos, sentimientos y procesos relacionados presentes en individuos con una amplia variedad de diagnósticos psiquiátricos (Swiller, 1988).

De acuerdo con Yaben y Montalvo (1994), Blanchard, Arena y Pallmeyer (1981) se presentaban tasas de entre 8 y $10 \%$ de alexitimia en población de estudiantes, con mayor presencia entre los hombres (8\% de varones alexitímicos frente al 1.8\% de mujeres). Asimismo, se ha encontrado una mayor presencia de alexitimia en individuos con un nivel socio-económico y educativo bajo (Cerezo, García-Moja, Gándara, \& Hernández, 1988).

La edad constituye también un elemento importante, dado que los individuos de mayor edad tienden a obtener puntuaciones más elevadas y puede explicarse por una reducción de la expresión espontánea (Páez \&Velasco, 1993).

Asimismo, los pacientes alexitímicos son significativamente más ansiosos y deprimidos que los no alexitímicos, y presentan un mayor número de quejas físicas y alteraciones psicológicas generales. El hecho de que esta característica se encuentre relacionada con una variedad de enfermedades psicosomáticas condujo a la asunción prematura y errónea de que existía una relación etiológica entre este rasgo y la enfermedad psicosomática. Sin embargo, se ha observado que muchos de los pacientes psicosomáticos no son alexitímicos, mientras otros, que sí lo son, no padecen enfermedades psicosomáticas, por lo que se ha concluido que ésta se presenta tanto en ambas poblaciones (Rodrigo \& Lusiardo, 1992).

Por otra parte, varios estudios confirman que la alexitimia es un rasgo estable presente en diversas culturas (Páez \& Casullo, 2000; Páez et al., 1999; Thorberg et al., 2016).

\section{Alexitimia y consumo de drogas}

La alexitimia es un rasgo común entre los consumidores de drogas (Morie et al., 2016), del cual desde hace tiempo han reportado tasas cercanas al 50\% (Taylor, Parker, \& Bagby, 1990; Taylor, Ryan, \& Bagby, 1985).

Sivak y Wiater (1997) señalan que son recurrentes los casos de personas alexitímicas con adicción al alcohol o a otras drogas que se encuentran en situaciones de estrés o de gran tensión, y que presentan un historial de molestias físicas.

Fernández-Serrano, Lozano Rojas, Pérez-García y Verdejo-García (2010) señalan la dificultad de los usuarios de drogas para reconocer emociones, en particular demostraron cómo los policonsumidores de sustancias tenían reconocimientos significativamente más pobres que los no consumidores de expresiones faciales como ira, asco, miedo y tristeza. También refieren que entre mayor era la cantidad y el lapso de consumo referidos, era menor la capacidad para reconocer emociones en general. Además, la dosis de cocaína consumida predijo un limitado reconocimiento de la ira, mientras que el mayor tiempo de consumo predijo un reconocimiento limitado tanto de la ira como del miedo.

En el mismo sentido, Hamidi, Rostami, Farhoodi y Abdolmanafi (2010) encontraron que los pacientes usuarios de drogas tienen más problemas y síntomas relacionados con la alexitimia en comparación con personas no consumidoras. Ghalehban y Besharat (2011) identificaron que los pacientes con trastorno de abuso de sustancias obtuvieron puntuaciones significativamente más altas que los no consumidores. De acuerdo con estos y otros autores (Morie et al., 2016) existe una relación entre la alexitimia y el abuso de sustancias en relación con la capacidad del individuo para distanciarse de las emociones negativas, automedicarse y facilitar la comunicación verbal y emocional.

De acuerdo con Moral de la Rubia (2009), los estudiantes alexitímicos tienden a deprimirse más, aunque no tengan una conciencia clara de su estado afectivo 
y tiendan a usar el alcohol como medio de afrontar ese malestar emocional confuso, Lyvers, Hasking, Albrecht y Thorberg (2012) reportan que el consumo de alcohol puede ser utilizado por personas con alexitimia para ayudar a hacer frente a la ansiedad u otro efecto negativo, así como para mejorar el desempeño social (Thorberg et al., 2016), estando presentes algunas diferencias por sexo (Luminet, de Sousa Uva, Fantini, \& de Timary, 2016).

Asimismo, Campanella et al. (2012) destacan la importancia de evaluar factores de la personalidad cuando se evalúan los niveles de alexitimia por sexo.

Lindsay y Ciarrochi (2009) señalan que la prevalencia de alexitimia medida mediante autoreporte en un grupo de consumidores de sustancias, recientemente abstinentes, fue del $50 \%$, que es superior de manera considerable a la que se observa entre la población general.

Casullo, Castro y D'Anna (2000) evaluaron a un grupo de 57 pacientes dependientes de sustancias psicoactivas en tratamiento hospitalario de comunidad terapéutica, con edad promedio de 24.5 años, y no encontraron perfiles psicopatológicos diferenciales entre los consumidores que son alexitímicos y los que no lo son, llegando a la conclusión de que la alexitimia es una alteración de los procesos comunicacionales, pero no está directamente vinculada con las manifestaciones psicopatológicas de la personalidad.

Evren, Evren, Dalbudak, Ozcelik y Oncu (2009) identificaron, en pacientes dependientes de sustancias que se encontraban en tratamiento hospitalario, que $45 \%$ presentaba alexitimia, ya que calificaba por arriba de 60 en el TAS-20 y que 57\% tenía al menos un tipo de antecedentes de violencia en la niñez. Asimismo, la tasa de desempleo, un bajo nivel de educación, el abuso emocional e historias de intentos de suicidio fueron más altos en los pacientes dependientes de sustancias alexitímicos. Además, los pacientes que tuvieron antecedentes de dos o más tipos de abuso o negligencia en la niñez también contaron con puntuaciones superiores a la media del TAS-20, en particular en el factor "dificultad para identificar emociones (DIE)". El número de tipos de situaciones traumáticas en la infancia correlacionó positivamente con el TAS-20 con los factores DIE y con la "dificultad para expresar las emociones (DEE)". Entre sus conclusiones se señala que el abuso emocional en la niñez podría ser un factor de riesgo para la alexitimia entre los dependientes de sustancias en tratamiento hospitalario. Lo anterior coincide con los resultados de Hahn, Simons y Simons (2016) que indican que las experiencias adversas en la niñez pueden afectar el funcionamiento emocional.

Por su parte, De Haan et al. (2013) refieren una relación entre una historia familiar de alcoholismo paterno y un mayor grado de alexitimia en pacientes usuarios de drogas, por lo que sugieren que esta podría ser una variable mediadora entre el uso de alcohol del padre y el consumo de sustancias por parte del sujeto.

También como variable mediadora, Spalletta, Troisi y Pasini (1995) evaluaron la presencia de alexitimia en un grupo de consumidores de cannabis recientemente abstinentes y encontraron diferencias en las tasas de alexitimia entre aquellos con y sin trastornos depresivos comórbidos. A partir de estos resultados, se sugiere que un síndrome diagnóstico de depresión mayor comórbida puede constituirse como una importante variable confusora en la evaluación de este rasgo.

Bonnet, Brèjard y Pedinielli (2013) realizaron análisis de mediación y validaron la hipótesis de que las dimensiones emocionales de la alexitimia actúan como mediadores entre la emocionalidad (emocionalidad negativa y la excitación emocional) y el uso de sustancias. Concluyen que como un factor de mediación, la alexitimia puede ser considerada como un tipo de proceso operacional que regula las emociones y que sus resultados podrían tener implicaciones importantes en intervenciones clínicas y terapéuticas enfocadas en estrategias de regulación de emociones y el consumo de sustancias.

Coriale et al. (2012) encontraron que los abusadores de alcohol alexitímicos consumen significativamente más alcohol y son menos abstinentes que los abusadores de alcohol no alexitímicos, encontrando también un importante efecto mediador de la alexitimia con respecto a las estrategias de afrontamiento para evitar el abuso del alcohol.

Por su parte, Aguilar de Arcos, Verdejo-García, Peralta-Ramírez, Sánchez-Barrera y Pérez-García (2005) señalan que los procesos emocionales puede encontrarse alterados de manera significativa en los usuarios de drogas y que estas alteraciones pueden comprometer la eficacia de un tratamiento. Casado (2012) indica que la presencia de síntomas de alexitimia en consumidores de drogas repercute negativamente en la posibilidad de realizar una gestión emocional efectiva.

Carroll (1999) encontró en consumidores de cocaína una tasa de alrededor del 39\% de personas que presentan alexitimia. Además, encontró que los pacientes con alexitimia responden menos a la terapia cognitiva conductual, pues dentro de este abordaje se necesita que los pacientes monitoreen, identifiquen y analicen sus ansiedades, estados afectivos negativos y cogniciones sutiles, lo que requiere de cierta capacidad introspectiva que representa una exigencia abrumadora para este tipo de pacientes.

Los estudios sobre alexitimia en México con usuarios de drogas son escasos, en particular destaca el de Corona Figueroa (2009) -realizado con pacientes de ClJ consumidores de cocaína de la región Centro de México-, 
cuyos resultados ponen de manifiesto la necesidad de establecer nuevas estrategias de acercamiento que permitan a los pacientes identificar dificultades relacionadas con la comunicación afectiva y la expresión de sus emociones.

Como se ha señalado, muchas de las estrategias de intervención en pacientes con consumo de drogas, entre las que se encuentran las terapias cognitivo conductuales, requieren una cierta capacidad introspectiva y de expresión que puede resultar abrumadora para ellos, de ahí el interés de determinar y comparar la presencia de este rasgo entre hombres y mujeres, usuarios de distintos tipos de droga, solicitantes de tratamiento, agrupados por sexo y droga de mayor impacto (en adelante DMI, esto es, la droga por la que solicitaron tratamiento), así como la influencia de la edad y el nivel de escolaridad en los niveles de alexitimia de los usuarios de las distintas drogas de estudio.

Esta información puede tener implicaciones importantes para el tratamiento y la rehabilitación del consumo de drogas.

\section{MÉTODO}

\section{Diseño}

Estudio transversal, comparativo, con muestra postestratificada por sexo y por droga de mayor impacto (DMI).

\section{Definición de variables}

\section{Alexitimia \\ Definición teórica}

La alexitimia es un constructo multifacético que incluye, por un lado, una dificultad para identificar sentimientos y estados emocionales subjetivos y distinguir entre los sentimientos y las sensaciones físicas producidas por las emociones; y, por otro, una dificultad para describir los propios sentimientos, así como una limitada capacidad para la fantasía y un estilo cognitivo orientado hacia el exterior (Nemiah, Freyberger Sifneos, 1976). De acuerdo con la definición de los factores obtenidos por Moral de la Rubia (2008), se considerarán como componentes de este constructo la "Dificultad para identificar emociones", la "Dificultad para expresar emociones" y el "Pensamiento externamente orientado".

\section{Definición operacional}

Operacionalmente se definirá el nivel de alexitimia como la suma total de los puntajes obtenidos en los tres fac- tores mencionados y se considerará como alexitimia a cualquier puntuación superior a 60 puntos obtenida con base en una escala de 20 reactivos tipo Likert, con cinco opciones de respuesta que van de uno a cinco; el rango de posibles resultados totales abarca de 20 a 100 puntos. La interpretación de los resultados se basará en los siguientes puntos de corte establecidos por Taylor y colaboradores (1992 y 2003):

$\leq 51$ puntos: Ausencia de alexitimia

De 52 a 60: Posible alexitimia

$\geq 61$ : Presencia de alexitimia

\section{Droga de mayor impacto}

Principal droga por la que se solicita tratamiento, tal como se registra en la Entrevista Inicial, según criterio del terapeuta.

\section{Instrumento}

Para el presente estudio se utilizó la Escala de Alexitimia de Toronto (TAS, por sus siglas en inglés) de Bagby, Parker y Taylor (1994), conformada por 20 reactivos, agrupados en tres factores (dificultad para identificar sentimientos, dificultad para expresar sentimientos y pensamiento externamente orientado), la cual ha sido ampliamente aplicada, incluyendo estudios realizados con usuarios de drogas.

EI TAS 20 se ha traducido a 18 diferentes lenguas y evaluado por medio de Análisis Factoriales Confirmatorios en 19 países, y en la mayoría se corrobora la estructura de tres factores de la escala (Taylor et al., 2003).

En México se ha validado la escala TAS por Pérez-Rincón, Cortés, Ortiz, Peña, Ruiz y Díaz-Martínez (1997), con población abierta y pacientes psiquiátricos no psicóticos atendidos en consulta externa; también por Páez et al. (1999) en el contexto de un estudio transcultural, con estudiantes de Psicología; y más recientemente, por Moral de la Rubia (2008), con estudiantes de Psicología de recién ingreso.

De acuerdo con la validación de Moral de la Rubia (2008) de la escala TAS-20, con estudiantes universitarios en México, la escala constituye un instrumento válido y confiable para valorar la presencia de alexitimia, siendo un instrumento más específico (95\%) que sensible (78\%) y con mayor capacidad para detectar la ausencia de rasgo que su presencia.

La escala completa presenta una alta consistencia interna (alfa de Cronbach de.81), en tanto que los tres factores que la integran tienen una consistencia entre adecuada y aceptable (.78, .75 y .66). 
Los tres factores de escala explican 31\% de varianza en forma global. Estos son: 1) Dificultad para la identificar emociones (ítems 1, 3, 6, 7, 9, 13 y 14), que explica $11.07 \%$; 2) Dificultad para expresar emociones (ítems 2 , 4, 11, 12 y 17), que explica $13.24 \%$; y 3) Pensamiento externamente orientado (ítems 5, 8, 10, 15, 16, 18, 19 y 20), que explica $6.35 \%$

En este estudio se aplicó una cédula con tres secciones. La primera indaga sobre características sociodemográficas (sexo, edad, estado civil y ocupación); la segunda explora el patrón de consumo de tabaco, alcohol, mariguana, cocaína, crack, inhalables, metanfetamina y heroína (alguna vez, en el último año y en el último mes), así como la droga por la que se solicita tratamiento (droga de mayor impacto); la tercera sección incluye la Escala de Alexitimia de Toronto, basada en las adaptaciones al español de Páez y Casullo (2000) y de Moral de la Rubia (2008), y para la cual se consideraron los puntos de corte establecidos por Taylor y colaboradores (1992 y 2003) señalados anteriormente.

\section{Muestra}

Con base en un muestreo no probabilístico, intencional y postestratificado por sexo y droga de mayor impacto, se conformó una muestra de 266 pacientes solicitantes de tratamiento en 25 unidades de atención para el consumo de drogas (CIJ) de distintos estados de la república mexicana, de los cuales 36 solicitaron tratamiento por consumo de alcohol, 46 por uso de cannabis, 29 por uso de cocaína, 41 por uso de crack, 30 por uso de inhalables, 53 por uso de heroína y 29 por uso de metanfetamina.

El porcentaje general de hombres fue de 76\% (199 personas) y el de mujeres de 24\% (64 personas). Más de la mitad $(61.8 \%)$ se encontraban solteros al momento de levantar la encuesta y $27 \%$ estaban casados o vivían en unión libre (13.0\% y 14.1\%, respectivamente), el resto estaba separado (9.2\%) o se había divorciado (1.9\%).
Respecto al nivel de escolaridad, casi $40.0 \%$ contaba con estudios de nivel bachillerato o superior y $35.7 \%$ con estudios de secundaria. Un porcentaje menor (17.3\%) había cursado la primaria y $1.6 \%$ no contaba con estudios.

En relación con la ocupación, destaca el alto porcentaje de los usuarios que se encontraba desempleado durante el levantamiento de la encuesta (24.2\%). Del resto de la muestra, más del $40.0 \%$ trabajaba, $16.4 \%$ estudiaba, $3.9 \%$ estudiaba y trabajaba y 13.7 se dedicaba al hogar.

\section{Análisis}

Se aplicó la prueba $t$ de Student para comparar las medias de los puntajes de alexitimia entre hombres y mujeres. No se realizaron comparaciones por sexo entre los distintos tipos de usuarios agrupados según DMI, ya que la postestratificación no permitió reunir a un suficiente número de mujeres para cada una de las drogas consideradas.

Por medio de un análisis de varianza de una sola vía ANOVA, se llevó a cabo una comparación de los tres factores que conforman la variable alexitimia por sexo. Asimismo, se efectuó un análisis multifactorial de la varianza MANOVA para comparar simultáneamente los tres factores al interior de los distintos grupos de usuarios agrupados por DMI. Dado que la literatura señala que la edad o el nivel de escolaridad pudiesen tener alguna influencia en la presencia de alexitimia, se realizaron pruebas de regresión lineal para explorar y corroborar el posible papel predictor de estas variables.

\section{RESULTADOS}

De acuerdo con los resultados, la mitad de los usuarios presenta rasgos de personalidad alexitímicos, una cuarta parte posiblemente los tiene y el resto no presenta dichos rasgos (Tabla 1).

Tabla 1

Diagnóstico de alexitimia en usuarios de drogas por sexo

\begin{tabular}{|c|c|c|c|c|c|c|}
\hline \multirow{2}{*}{ Diagnóstico } & \multirow{2}{*}{$N$} & \multirow{2}{*}{$\%$} & \multicolumn{2}{|c|}{ Hombres } & \multicolumn{2}{|c|}{ Mujeres } \\
\hline & & & $n$ & $\%$ & $n$ & $\%$ \\
\hline Ausencia & 63 & 23.9 & 46 & 23.0 & 21 & 32.8 \\
\hline Posible & 65 & 24.6 & 52 & 26.0 & 13 & 20.3 \\
\hline Presencia & 136 & 51.5 & 102 & 51.0 & 30 & 46.9 \\
\hline Total & 264 & 100.0 & 200 & 100.0 & 64 & 100.0 \\
\hline
\end{tabular}


Con respecto a los análisis por sexo, no se encontraron diferencias estadísticamente significativas en la puntuación general de alexitimia entre hombres $(\bar{x}=61.1$, $\mathrm{DE}=13.3)$ y mujeres $(\bar{x}=59.2, \mathrm{DE}=16.1)$.

Sin embargo, en los análisis realizados al interior de los factores se encontraron diferencias significativas entre ambos sexos en el factor $1(t=1.994, g l=261, p=.047)$, es decir, en la dificultad para identificar emociones; indica que los hombres presentan mayor dificultad que las mujeres para identificar dichas emociones (hombres: $\bar{x}=20.7$, $\mathrm{DE}=6.8$; y mujeres: $\bar{x}=18.7, \mathrm{DE}=7.2$ ).

Las pruebas de análisis de varianza (ANOVA) no mostraron diferencias estadísticamente significativas en las puntuaciones de alexitimia por droga de mayor impacto ( $F=.537, g l=6, p=.780$; Tabla 2).

El análisis multifactorial de la varianza MANOVA, mediante el cual se compararon simultáneamente los tres factores entre los distintos grupos de usuarios según DMI, no arrojó diferencias estadísticamente significativas ( $F=1.240, p=.222)$. Tampoco se observaron diferencias entre los grupos al comparar cada factor en forma separada (factor $1: F=.528, g l=6, p=.787$; factor $2: F=.711$, $g l=6, p=.641$; factor $3: F=1.443, g l=6, p=.199)$, como se muestra en la Tabla 3.

Por otra parte, mediante un conjunto de análisis de regresión lineal múltiple para cada uno de los grupos según su droga de mayor impacto (Tabla 4), se pudo observar que la edad sólo es un predictor significativo del incremento de alexitimia entre los usuarios de metanfetamina $(\beta=.445 ; p<.05)$ y la escolaridad lo es únicamente para los usuarios de $\operatorname{crack}(\beta=.445 ; p<.05)$.

\section{DISCUSIÓN}

Los resultados obtenidos permiten corroborar que tal como se señala en diversos estudios (Corona Figueroa,
2009; Evren et al., 2009; Ghalehban \& Besharat, 2011; Hamidi et al. 2010; Lindsay \& Ciarrochi, 2009; Taylor, Parker \& Bagby, 1990) la presencia de alexitimia es considerablemente mayor en usuarios de drogas que entre la población general, con una diferencia de alrededor de 40 puntos porcentuales entre una y otra.

Resulta relevante el hecho de que no se observen diferencias significativas en las puntuaciones de alexitimia entre los usuarios de las distintas drogas consideradas, ni en la escala general ni en los tres factores que la conforman.

Con relación al sexo de los participantes, destaca que, en contra de lo esperado, no se observen diferencias entre los usuarios hombres y mujeres en la puntuación general de alexitimia, mientras que la literatura en la materia (Blanchard et al., 1981; Yaben \& Montalvo, 1994) refiere que los hombres tienden a presentar puntuaciones bastante más altas de alexitimia que las mujeres en la población general (8.1 en hombres frente a 1.8 en mujeres), lo que sugiere que entre los usuarios de drogas estas diferencias se diluyen; la excepción es el caso del factor uno, relativo a la dificultad para identificar emociones, en el que los hombres obtuvieron puntajes ligeramente superiores al de las mujeres (20.7 contra 18.7).

Lo anterior es relevante si se considera que los pacientes con alexitimia presentan dificultades para responder a la terapia de tipo cognitivo conductual, dada la menor capacidad para identificar y expresar emociones (Carroll, 1999), sobre todo teniendo en cuenta que se trata de un abordaje ampliamente utilizado en el tratamiento de adicciones. Sería entonces conveniente trabajar, como lo propone Corona Figueroa (2009), en el desarrollo de estrategias que favorezcan la capacidad introspectiva de los pacientes, de tal modo que les sea posible reconocer, analizar y expresar sus emociones.

Tabla 2

Alexitimia en usuarios de distintas drogas por sexo

\begin{tabular}{|c|c|c|c|c|c|c|c|}
\hline \multirow{2}{*}{$\begin{array}{l}\text { Droga de Mayor } \\
\text { Impacto }\end{array}$} & \multirow{2}{*}{$n$} & \multirow{2}{*}{$\bar{x}$} & \multirow{2}{*}{$D E$} & \multicolumn{2}{|c|}{ Hombres } & \multicolumn{2}{|c|}{ Mujeres } \\
\hline & & & & $\bar{x}$ & $D E$ & $\bar{x}$ & $D E$ \\
\hline Alcohol & 36 & 61.0 & 14.9 & 57.6 & 12.2 & 67.7 & 18.0 \\
\hline Cannabis & 46 & 61.2 & 14.6 & 61.5 & 15.8 & 60.2 & 10.6 \\
\hline Cocaína & 29 & 56.9 & 12.8 & 59.5 & 11.7 & 45.2 & 11.0 \\
\hline Crack & 41 & 61.6 & 14.7 & 61.3 & 13.4 & 62.1 & 18.1 \\
\hline Heroína & 53 & 61.8 & 12.4 & 61.6 & 12.9 & 64.0 & 4.8 \\
\hline Inhalables & 30 & 58.9 & 14.3 & 63.3 & 12.7 & 48.7 & 13.0 \\
\hline Metanfetamina & 29 & 61.4 & 15.1 & 61.8 & 14.0 & 60.7 & 17.7 \\
\hline Global & 264 & 60.6 & 14.0 & 61.1 & 13.3 & 59.2 & 16.1 \\
\hline
\end{tabular}


Tabla 3

Factores componentes de la alexitimia en usuarios de distintas drogas por sexo

\begin{tabular}{|c|c|c|c|c|c|c|c|c|}
\hline & \multirow{2}{*}{$\begin{array}{c}\text { Droga de Mayor } \\
\text { Impacto }\end{array}$} & \multirow{2}{*}{$n$} & \multirow{2}{*}{$\bar{x}$} & \multirow{2}{*}{$D E$} & \multicolumn{2}{|c|}{ Hombres } & \multicolumn{2}{|c|}{ Mujeres } \\
\hline & & & & & $\bar{x}$ & $D E$ & $\overline{\bar{x}}$ & $D E$ \\
\hline \multirow{8}{*}{ 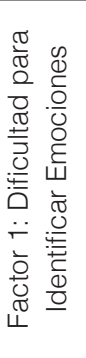 } & Alcohol & 36 & 19.2 & 8.2 & 18.0 & 7.4 & 21.7 & 9.5 \\
\hline & Cannabis & 46 & 21.4 & 7.3 & 22.3 & 7.7 & 18.8 & 5.2 \\
\hline & Cocaína & 29 & 19.2 & 6.7 & 20.4 & 5.9 & 13.3 & 6.6 \\
\hline & Crack & 41 & 20.0 & 6.3 & 20.8 & 6.0 & 18.0 & 6.6 \\
\hline & Heroína & 53 & 20.2 & 6.4 & 20.2 & 6.6 & 20.0 & 4.1 \\
\hline & Inhalables & 30 & 20.2 & 6.8 & 22.0 & 6.2 & 15.9 & 6.4 \\
\hline & Metanfetamina & 29 & 20.9 & 6.9 & 20.8 & 6.8 & 21.1 & 7.7 \\
\hline & Global $^{*}$ & 264 & 20.2 & 6.9 & 20.7 & 6.8 & 18.7 & 7.2 \\
\hline \multirow{8}{*}{ 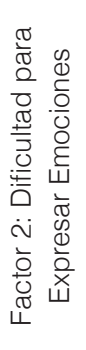 } & Alcohol & 36 & 14.4 & 5.1 & 13.6 & 5.2 & 15.8 & 4.7 \\
\hline & Cannabis & 46 & 13.7 & 5.2 & 13.7 & 5.4 & 13.7 & 4.5 \\
\hline & Cocaína & 29 & 13.6 & 4.0 & 14.4 & 3.6 & 10.3 & 4.2 \\
\hline & Crack & 41 & 14.9 & 5.1 & 14.7 & 4.4 & 15.6 & 6.7 \\
\hline & Heroína & 53 & 15.1 & 4.6 & 15.0 & 4.7 & 17.3 & 3.0 \\
\hline & Inhalables & 30 & 13.8 & 4.4 & 15.0 & 4.3 & 11.0 & 3.5 \\
\hline & Metanfetamina & 29 & 14.8 & 4.8 & 15.0 & 4.6 & 14.4 & 5.4 \\
\hline & Global & 264 & 14.4 & 4.8 & 14.5 & 4.7 & 14.1 & 5.2 \\
\hline \multirow{8}{*}{ 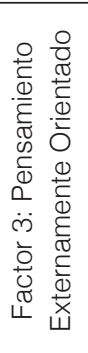 } & Alcohol & 36 & 27.4 & 4.8 & 26.0 & 3.6 & 30.2 & 5.7 \\
\hline & Cannabis & 46 & 26.0 & 5.4 & 25.5 & 5.9 & 27.6 & 3.6 \\
\hline & Cocaína & 29 & 24.1 & 4.2 & 24.8 & 4.3 & 21.5 & 3.3 \\
\hline & Crack & 41 & 26.6 & 6.0 & 25.9 & 5.2 & 28.5 & 7.5 \\
\hline & Heroína & 53 & 26.4 & 4.0 & 26.4 & 4.1 & 26.8 & 3.6 \\
\hline & Inhalables & 30 & 25.0 & 5.6 & 26.3 & 5.3 & 21.8 & 5.5 \\
\hline & Metanfetamina & 29 & 25.7 & 5.9 & 26.0 & 5.3 & 25.2 & 7.1 \\
\hline & Global & 264 & 26.0 & 5.2 & 25.9 & 4.8 & 26.4 & 6.3 \\
\hline
\end{tabular}

$* p<.05$

Tabla 4

Regresión lineal múltiple de la edad y el nivel de escolaridad como predictores de alexitimia en usuarios de distintas drogas

\begin{tabular}{|c|c|c|c|c|c|c|}
\hline $\begin{array}{l}\text { Droga de Mayor } \\
\text { Impacto }\end{array}$ & $R^{2}$ & $\begin{array}{c}\text { Variables } \\
\text { antecedentes }\end{array}$ & $t$ & $g l$ & $\beta$ & Sig. \\
\hline \multirow{2}{*}{ Alcohol } & \multirow{2}{*}{.065} & Edad & -1.609 & 2 & -.268 & N.S. \\
\hline & & Escolaridad & 1.210 & 2 & .201 & N.S. \\
\hline \multirow{2}{*}{ Cannabis } & \multirow{2}{*}{.004} & Edad & .245 & 2 & .037 & N.S. \\
\hline & & Escolaridad & 1.479 & 2 & .221 & N.S. \\
\hline \multirow{2}{*}{ Cocaína } & \multirow{2}{*}{-.043} & Edad & -.497 & 2 & -.102 & N.S. \\
\hline & & Escolaridad & -.800 & 2 & -.164 & N.S. \\
\hline \multirow{2}{*}{ Crack } & \multirow{2}{*}{.103} & Edad & .926 & 2 & .151 & N.S. \\
\hline & & Escolaridad & 2.454 & 2 & .400 & $<.05$ \\
\hline \multirow{2}{*}{ Heroína } & \multirow{2}{*}{.011} & Edad & .067 & 2 & .469 & N.S. \\
\hline & & Escolaridad & .081 & 2 & .561 & N.S. \\
\hline \multirow{2}{*}{ Inhalables } & \multirow{2}{*}{.008} & Edad & -.082 & 2 & -.016 & N.S. \\
\hline & & Escolaridad & .469 & 2 & .090 & N.S. \\
\hline \multirow{2}{*}{ Metanfetamina } & \multirow{2}{*}{.133} & Edad & 2.387 & 2 & .445 & $<.05$ \\
\hline & & Escolaridad & .376 & 2 & .070 & N.S. \\
\hline
\end{tabular}


Cabe señalar que una de las limitaciones del presente trabajo es que los resultados sólo son generalizables a los usuarios en tratamiento y que el tamaño de muestra de las mujeres no es suficiente para realizar análisis comparativos por sexo y tipo de sustancia, por lo cual resultaría conveniente que en futuros estudios se considere la captación de un mayor número de mujeres, con el fin de realizar tales comparaciones, sobre todo en el caso de las personas consumidoras de alcohol, cocaína e inhalables.

Si bien el presente estudio cumple con el objetivo propuesto de explorar la presencia de alexitimia entre usuarios de drogas que asisten a solicitar tratamiento, sería importante indagar más adelante la existencia de antecedentes de violencia y maltrato durante la infancia (Hahn et al., 2016) y de abuso o dependencia de alcohol por parte del padre (De Haan et al., 2013), así como la presencia de síntomas de ansiedad y depresión (Campanella et al., 2012; Lyvers et al., 2012), variables vinculadas de manera significativa con la alexitimia y el consumo de drogas, según se reporta en la literatura sobre la materia.

\section{CONFLICTOS DE INTERÉS}

Aun cuando los autores laboran en Centros de Integración Juvenil, A.C., el contenido del presente artículo representa la opinión de estos y no de la institución.

\section{REFERENCIAS}

Aguilar de Arcos, F., Verdejo-García, A., Peralta-Ramírez, M. I., Sánchez-Barrera, M., \& Pérez-García, M. (2005). Experience of emotions in substance abusers exposed to images containing neutral, positive, and negative affective stimuli. Drug and Alcohol Dependence, 78(2), 159-167.

Alonso-Fernández, F. (2011). La alexitimia y su trascendencia clínica y social. Salud Mental, 34(6), 481-490.

Bagby, R. M., Parker, J. D. A., \& Taylor, G. J. (1994). The twenty-item Toronto alexithymia scale-I. Item selection and cross-validation of the factor structure. Journal of Psychosomatic Research, 38(1), 23-32.

Blanchard, E. B., Arena. J. G., \& Pallmeyer, T. P. (1981). Psychometric properties of a scale to measure alexithymia. Psychotherapy and Psychosomatics, 35(1), 64-71.

Bonnet, A., Brèjard, V., \& Pedinielli, J. L. (2013). Emotional dispositions and substance use: Mediating effect of alexithymia. Psychological Reports, 112(1), 289-302.

Campanella, S., Falbo, L., Rossignol, M., Grynberg, D., Balconi, M., Verbanck, P., ... Maurage, P. (2012). Sex differences on emotional processing are modulated by subclinical levels of alexithymia and depression: A preliminary assessment using event-related potentials. Psychiatry Research, 197(1), 145-153.
Carroll, K. M. (1999). Manual I. Tratamiento de la adicción a la cocaína: Un enfoque cognitivo-conductual. Estados Unidos: National Institute on Drug Abuse.

Casado, D. C. (2012). Gestión emocional como factor de protección frente al consumo de drogas en el ámbito laboral. Aprende $\mathrm{RH}$ : La revista de los recursos humanos y del e-learning, (36), 90-94.

Casullo, M. M., Castro, A., \& D’Anna, A. (2000). Alexitimia y características de personalidad de jóvenes adictos. En D. Páez \& M. M. Casullo (Eds.), Cultura y alexitimia (pp. 181-192). Buenos Aires: Paidós.

Cerezo, P., García-Moja, C., Gándara, J. J., \& Hernández, H. (1988). Aspectos teóricos, clínicos y evaluación de la alexitimia. Psiquis, 9(6-7), 19-29.

Coriale, G., Bilotta, E., Leone, L., Cosimi, F., Porrari, R., De Rosa, F., Ceccanti, M. (2012). Avoidance coping strategies, alexithymia and alcohol abuse: A mediation analysis. Addictive Behaviors, 37(11), 1224-1229.

Corona Figueroa, B. A. (2009) Evaluación psicométrica de medidas de identificación y expresión de emociones en usuarios de cocaína atendidos en los Centros de Integración Juvenil de la zona metropolitana de Guadalajara. (Tesis de maestría no publicada). Universidad de Guadalajara, México.

De Haan, H. A., Joosten, E. A., De Haan, L., Schellekens, A. F., Buitelaar, J. K., van der Palen, J., ... De Jong, C. A. (2013). A family history of alcoholism relates to alexithymia in substance use disorder patients. Comprehensive Psychiatry, 54(7), 911-917.

Evren, C., Evren, B., Dalbudak, E., Ozcelik, B., \& Oncu, F. (2009). Childhood abuse and neglect as a risk factor for alexithymia in adult male substance dependent inpatients. Journal of Psychoactive Drugs, 41(1), 85-92.

Fernández-Serrano, M. J., Lozano Rojas, O., Pérez-García, M., Verdejo-García, A. (2010). Impact of severity of drug use on discrete emotions recognition in polysubstance abusers. Drug and Alcohol Dependence, 109, 57-64.

Gabriel, B., Untas, A., Lavner, J. A., Koleck, M., \& Luminet, O. (2016). Gender typical patterns and the link between alexithymia, dyadic coping and psychological symptoms. Personality and Individual Differences, 96, 266-271.

Ghalehban, M., \& Besharat, M. A. (2011). Examination and comparison of alexithymia and self-regulation in patients with substance abuse disorder and normal individuals. Procedia Social and Behavioral Sciences, 30, 38-42.

Günther, V., Matthes, A., Kersting, A., Egloff, B., \& Suslow, T. (2016). Alexithymia and the implicit self-concept of extraversion in women. Personality and Individual Differences, 88, 21-25.

Hahn, A. M., Simons, R. M., \& Simons, J. S. (2016). Childhood maltreatment and sexual risk taking: The mediating role of alexithymia. Archives of Sexual Behavior, 45(1), 53-62.

Hamidi, S., Rostami, R., Farhoodi, F., \& Abdolmanafi, A. (2010). A study and comparison of Alexithymia among patients with substance use disorder and normal people. Procedia Social and Behavioral Sciences, 5, 1367-1370. 
Levant, R. F., Hall, R. J., Williams, C. M., \& Hasan, N. T. (2009). Gender differences in alexithymia. Psychology of Men \& Masculinity, 10(3), 190.

Lindsay, J., \& Ciarrochi, J. (2009). Substance abusers report being more alexithymic than others but do not show emotional processing deficits on a performance measure of alexithymia. Addiction Research \& Theory, 17(3), 315-321.

Luminet, O., de Sousa Uva, M. C., Fantini, C., \& de Timary, P. (2016). The association between depression and craving in alcohol dependency is moderated by gender and by alexithymia factors. Psychiatry Research, 239, 28-38.

Lyvers, M., Hasking, P., Albrecht, B., \& Thorberg, F. A. (2012). Alexithymia and alcohol:The roles of punishment sensitivity and drinking motives. Addiction Research \& Theory, 20(4), 348-357.

Moral de la Rubia, J. (2009). Alexitimia y rasgos de alcoholismo, una relación mediada por la depresión. Medicina Universitaria, 11(43), 99-108.

Moral de la Rubia, J. (2008). Propiedades psicométricas de la escala de Alexitimia de Toronto de 20 reactivos en México. Revista Electrónica de Psicología Iztacala, 11(2). Recuperado de: http://campus.iztacala.unam.mx/carreras/psicologia/psiclin/ vol11num2/art5-vol11-No2.pdf

Morie, K. P., Yip, S. W., Nich, C., Hunkele, K., Carroll, K. M., \& Potenza, M. N. (2016). Alexithymia and addiction: A review and preliminary data suggesting neurobiological links to reward/loss processing. Current Addiction Reports, 3(2), 239-248.

Nemiah, J. C., Freyberger, H., \& Sifneos, P. E. (1976). Alexithymia: A view of the psychosomatic process. Modern trends in psychosomatic medicine, 3, 430-439.

Páez, D., \& Casullo, M. M. (2000). Presentación de las propiedades psicométricas de la Escala de Alexitimia de Toronto (TAS-20) en comunidades de habla hispana y otros países. En D. Páez, \& M. M. Casullo (Eds.), Cultura y alexitimia (pp. 199-203). Buenos Aires: Paidós.

Páez, D., Martínez-Sánchez, F., Velasco, C., Mayordomo, S., Fernández, I., \& Blanco, A. (1999). Validez psicométrica de la Escala de Alexitimia de Toronto (TAS-20): Un estudio transcultural. Boletín de Psicología, 63, 55-76.

Páez, D., \& Velasco, C. (1993). Alexitimia: una revisión de los conceptos, de los instrumentos y una comparación con la represión. En D. Páez (Ed.), Salud, expresión y represión social de las emociones. Valencia: Promolibro.
Pérez-Rincón, H., Cortés, J., Ortíz, S., Peña, J., Ruiz, J., \& Díaz-Martínez, A. (1997). Validación y estandarización de la versión española de la Escala Modificada de Alexitimia de Toronto. Salud mental, 20(3), 30-34.

Rodrigo, G., \& Lusiardo, M. (1992). Factor structure of a Spanish version of the Toronto Alexithymia Scale. Psychotherapy and psychosomatics, 58(3-4), 197-201.

Sifneos, P. E. (1973). The prevalence of alexithymic characteristics in psychosomatic patients. Psychotherapy and Psychosomatics, 22, 255-262.

Sivak, R., \& Wiater, A. (1997). Alexitimia, la dificultad para verbalizar afectos. Teoría y clínica. Buenos Aires: Paidós.

Spalletta, G., Troisi, A., \& Pasini, A. (1995). Alextthymia in male cannabis users: The role of comorbid depressive disorders. Depression, 3(5), 246-249.

Swiller, H. I. (1988). Alexithymia: Treatment utilizing combined individual and group psychotherapy. International Journal of Group Psychotherapy, 38(1), 47-61.

Taylor, G. J., Bagby, R., \& Parker, J. D. (2003). The 20-Item Toronto Alexithymia Scale: IV. Reliability and factorial validity in different languages and cultures. Journal of psychosomatic research, 55(3), 277-283.

Taylor, G. J., Bagby, R.M. \& Parker, J. D. A. (1997). Disorders of affect regulation: Alexithymia in medical and psychiatric illness. Cambridge, MA: Cambridge University Press.

Taylor, G. J., Bagby, R. M., \& Parker J. D. A. (1992). The revised Toronto Scale: Some reliability, validity and normative data. Psychotherapy and Psychosomatics, 57(1-2), 34-41.

Taylor, G. J., Parker, J. D., \& Bagby, R. M. (1990). A preliminary investigation of alexithymia in men with psychoactive substance dependence. The American Journal of Psychiatry, 147(9), 1228.

Taylor, G. J., Ryan, D. P., \& Bagby, R. M. (1985). Toward the development of a new self-report alexithymia scale. Psychotherapy and Psychosomatics, 44, 191-199.

Thorberg, F. A., Young, R. M., Sullivan, K. A., Lyvers, M., Hurst, C. P., Connor, J. P., ... Feeney, G. F. (2016). A longitudinal mediational study on the stability of alexithymia among alcohol-dependent outpatients in cognitive-behavioral therapy. Psychology of Addictive Behaviors, 30(1), 64.

Yaben, S. Y., \& Montalvo, J. F. (1994). Alexitimia: Concepto, evaluación y tratamiento. Psicothema, 6(3), 357-366. 